\title{
A CRITICAL STUDY ABOUT THE TOXICITY OF LEAD AND THE PRECAUTIONARY MEASURES: A REVIEW
}

\author{
ARTI GUPTA ${ }^{\mathrm{a}}$, NISREEN HUSSAIN ${ }^{\mathrm{b}}$ AND BHAWANA PANDEY ${ }^{\mathrm{c}}$ \\ ${ }^{a}$ Department of Chemistry, Govt. Dr. W.W.P. Girl's P.G. College, Durg, Chhattisgarh, India \\ ${ }^{\text {b}}$ Department of Zoology, Govt. Dr. W.W.P. Girl's P.G. College, Durg, Chhattisgarh, India \\ 'Department of Biotechnology \& Microbiology, Bhilai Mahila Mahavidyalaya, Hospital Sector, Bhilai, Chhattisgarh, India
}

\begin{abstract}
Lead is the most important toxic heavy element in the environment. Due to its important physico-chemical properties, its use are quite remarkable. Globally it is an abundantly distributed, important yet dangerous environmental chemical. It's important properties like softness, malleability, ductility, poor conductibility and resistance to corrosion seem to make difficult to give up its use. Due to its non-biodegradable nature and continuous use, its concentration accumulates in the environment with increasing hazards.
\end{abstract}

KEYWORDS: Toxic Heavy Element, Malleability, Ductility, Non-Biodegradable Nature

Human exposure to lead and its compounds occurs mostly in lead related occupations with various sources like leaded gasoline, industrial processes such as smelting of lead and its combustion, pottery, boat building, lead based painting, lead containing pipes, battery recycling, grids, arm industry, pigments, printing of books, etc. Though its widespread use has discontinued in many countries of the world, it is still used in many industries like car repair, battery manufacturing and recycling, refining, smelting, etc. Lead is a highly poisonous metal affecting almost every organ in the body. Of all the organs, the nervous system is the mostly affected target in lead toxicity, both in children and adults. The toxicity in children is however of a greater impact than in adults. This is because their tissues, internal as well as external, are softer than in adults. Long-term exposure of adults can result in activities that measure functions of the nervous system. Infants and young children are especially sensitive to even low levels of lead, which may contribute to behavioral problems, learning deficits and lowered IQ. Long-time exposure to lead has been reported to cause anaemia, and blood pressure, mainly in old and middle aged people. Severe damage to the brain and kidneys, both in adults and children, were found to be linked to exposure to heavy lead levels resulting in death.

\section{WHAT IS TOXICITY}

Toxicity is the degree to which a chemical substance or a particular mixture of substances can damage an organism. Toxicity can refer to the effect on a whole organism, such as an animal, bacterium, or plant, as well as the effect on a substructure of the organism, such as a cell (cytotoxicity) or an organ such as the liver (hepatotoxicity).

Sometimes the word is more or less synonymous with poisoning in everyday usage. A central concept of toxicology is that the effects of a toxin are dose-dependent; even water can lead to water intoxication when taken in too high a dose, whereas for even a very toxic substance such as snake venom there is a dose below which there is no detectable toxic effect. Toxicity is species-specific, making cross-species analysis problematic. Newer paradigms and metrics are evolving to bypass animal testing, while maintaining the concept of toxicity endpoints. Toxic effects holder importance when plants, animals and human beings are considered, along with their surrounding environment.

\section{TYPES OF TOXICITY}

There are generally four types of toxic entities, Vis.; chemical, biological, physical and radiation:

- Chemical Toxicants include inorganic substances such as, lead, mercury, hydrofluoric acid, and chlorine gas, and organic compounds such as methyl alcohol, most medications, and poisons from living things. While some weakly radio active substances, such as uranium, are also chemical toxicants, more strongly radioactive materials like radium are not, their harmful effects (radiation poisoning) being caused by the ionizing radiation produced by the substance rather than chemical interactions with the substance itself.

- Disease-causing microorganisms and parasites are toxic in a broad sense, but are generally called

${ }^{1}$ Corresponding author 
pathogens rather than toxicants. The Biological Toxicity of pathogens can be difficult to measure because the "threshold dose" may be a single organism. Theoretically one virus, bacterium or worm can reproduce to cause a serious infection. However, in a host with an intact immune system the inherent toxicity: of the organism is balanced by the host's ability to fight back. Nonliving biological toxicants are generally called toxins if produced by a microorganism, plant, or fungus, and venoms if produced by an animal.

- Physical Toxicants are substances that, due to their physical nature, interfere with biological processes. Examples include coal dust, asbestos fibers or finely divided silicon dioxide, all of which can ultimately be fatal if inhaled. Corrosive chemicals possess physical toxicity because they destroy tissues, but they're not directly poisonous unless they interfere directly with biological activity. Water can act as a physical toxicant if taken in extremely high doses because the concentration of vital ions decreases dramatically if there's too much water in the body. Asphyxiate gases can be considered physical toxicants because they act by displacing oxygen in the environment but they are inert, not chemically toxic gases.

\section{TOXICITY OF ELEMENT LEAD}

Several methods are used to detect elevated blood lead levels. The presence of changes in blood cells visible under the microscope or deletion of dense lines in the bones of children seen on X-ray are signs used for detecting lead poisoning. However the main tool to detect elevated levels of body lead is to measure the level of lead in blood samples. This test gives however only an account of lead present in circulating blood but cannot show how much lead is stored in the body. As of 2012, the Centers for Disease Control and Prevention (USA) have set the standard elevated blood lead level for adults to be $10 \mu \mathrm{g} / \mathrm{dL}$ and for children $5 \mu \mathrm{g} / \mathrm{dL}$ of the whole blood. Previously, the standard lead level for children was $10 \mu \mathrm{g} / \mathrm{dL}$. The appearance of clinical manifestations varies from individual to individual depending on other environmental factors. In some there is a clear appearance of clinical features even at lower levels, while some are asymptomatic even at higher levels of lead present in their body fluids. Children are more prone to the effects of lead because usually their organs are in a developing stage. Thus blood lead levels have to be set lower and must be frequently checked, particularly where contamination is expected.

\section{HARMFUL EFFECTS ON FOOD, HEALTH AND ENVIRONMENT}

All along human history, lead poisoning has been reported to have severe effects. Occasional lead poisoning was found to be caused by lead salts used in pottery glazes leached by acidic fruit juices. It is also assumed that in the eighteenth and early nineteenth century lead was illegally added to wine both as a sweetener and to make it appear fresh. Lead poisoning is believed to be primarily responsible for the collapse of the Roman Empire, in which lead acetate was used as a sweetener of wine. Its prolonged use was considered to have caused dementia to many Roman emperors. Lead poisoning has, also been found to be the cause of anaemia in a number of cases as lead inhibits porphobilinogen synthase and ferrochelatase, preventing both porphobilinogen formation and the incorporation of iron into protoporphyrin IX, which prevents heme synthesis. One of the mechanisms by which lead interferes with cognition is that it acts as calcium analogue which interferes with ion channels. It has been observed that $\mathrm{Pb}^{2+}$ is a potent reversible and selective: blocker of voltage-dependent calcium channels at low concentrations. It has been known that the toxic effects on blood cells of rates caused by lead nitrate was alleviated by sodium selenite. They also showed that effects of lead nitrate were more harmful in diabetic than in non-diabetic rats. Oxidative stress was studied by low level lead exposure in first grade Uruguayan children, suggesting its lead has potentially adverse effects on oxidative stress.

Impaired respiratory function was observed in workers exposed to lead with elevated blood lead concentration and zinc protopbrphyrin concentration.

\section{PRECAUTIONARY MEASURES}

Lead poisoning causes severe effects and is a matter of serious concern, yet importantly, it is preventable. The best approach is to avoid exposure to lead it is recommended to frequently wash the children's hands and also recommended to discourage children from putting their hands, which can be contaminated, in their mouth habitually, thus increasing the chances of getting poisoned by lead. Vacuuming frequently and eliminating the use and 
or presence of lead containing objects like blinds and jewellery in the house can also help to prevent exposures. House pipes containing lead or plumbing solder fitted in old houses should be replaced to avoid lead contamination through drinking water. It is believed that hot water contains higher lead levels than does cold water, so it is recommended that for household uses cold water should be preferred to hot water. Dimercaprol and succimer constitute the treatment process for lead poisoning.

Lead poisoning is generally treated by using chelating salt disodium calcium edentate, which is the calcium chelate of the disodium salt of ethylene-diaminetetracetic acid (EDTA). Such chelating agents have a great affinity to the removing agent. The chelating agent for lead has a greater affinity to lead than calcium and so the lead chelate is formed by exchange. This is then excreted in urine, leaving behind harmless calcium. Blood lead levels were shown to be lowered by treatment with succimer used as chelation therapy in children exposed to lead to improve their neuropsychological development. And yet, though succimer was observed to help in reducing blood lead levels, it failed in improving the scores of cognition tests (Rogan et al., 2001). There is a number of antioxidants which are believed to act against toxicity of chemicals like lead and its related compounds. A new technique called nano-encapsulation of antioxidants may provide improved biodistribution and bioavailability of poorly soluble therapeutics through solubilisation (Flora et al., 2012).

Encapsulation of curcumin in a pluronic block copolymer demonstrated a slow and sustained release of curcumin and showed anticancer activity comparable with free curcumin (Sahu et al., 2010). These new techniques may hold a promise for treating a number of human diseases. In a very recent study, it was observed that puerarin promoted Akt and GSK-3J3 phosphorylation in PC12 cells exposed to lead acetate. The authors of the study concluded that puerarin as a phytoestrogen might be an attractive agent for prevention and treatment of chronic diseases related to lead neurotoxicity. In another recent finding beta-carotene was observed to have an antioxidant action and exert some beneficial effects in lead poisoning, independent of chelation (Dobrkowski et al., 2014). The authors also found significantly decreased homocysteine levels due to administration of betacarotene in lead exposed workers. Recently a study on a group of workers occupationally exposed to lead found that those treated with
$\mathrm{N}$-acetylcysteine (NAC) showed a significant reduction in their blood lead levels. In addition, all groups receiving NAC were shown to have significantly elevated activity of glutamate dehydrogenase. It was further reported that treatment with NAC normalised the level of homocysteine and decreased oxidative stress. It was thus concluded that NAC could be recommended as an alternative therapy for chronic lead toxicity in humans.

\section{DISCUSSION AND CONCLUSION}

Of all the heavy metal poisonings, lead poisoning appears to be rather prominent. The use of lead has been evidenced from ancient times and its toxicity reports are well documented. Due to its important physico-chemical properties, it has been used all over the world. With the onset on industrialization from the seventeenth century onwards, its use increased manifold, leading to increased toxicity in humans. Children are at a higher risk, particularly at sites where lead related occupations are nearby their playing grounds. Workers who are occupationally exposed to lead are also at increased risk of lead poisoning. Children of parents who are occupationally exposed to lead should be frequently checked for lead levels in their blood to avoid lead related risks. Lead toxicity is evident from the literature and there is almost no function in the body which is not affected by lead. Lead toxicity disrupts the functions of the digestive system, nervous system, respiratory system, reproductive system, etc. In addition, lead prevents enzymes from performing their normal activities. Lead even disrupts the normal DNA transcription process and causes disability in bones. Lead as such has no physiological role in the body and even smaller levels of lead can cause toxicity. The good news is however that it can be reversed and the levels of lead can be reduced from the body by a number of techniques used nowadays. The prominent ones among them are chelation therapy, nano-encapsulation, $\mathrm{N}$-acetylcysteine (NAC). A number of antioxidants also help in the removal of lead from the body. Though there are several methods of treatment available nowadays, it is certainly better to prevent direct exposure to toxins and thus preclude future consequences. It is also recommended that parents should educate their children about how to prevent accidental lead poisoning. The treatment strategies are not equally effective for everybody due to the differences ranging from genetic factors to environment and diet. 


\section{REFERENCES}

Advisory Committee on Childhood Lead Poisoning Prevention (ACCLPP)", Sinks T. Lead Exposures in U.S. Children, 2008. Implications for Prevention. [PMC free article] [Pub. Med]; Rubin $\mathrm{R}$, Strayer DS.

https://en.wikipedia.org/wiki/Lead_poisoning Lead poisoning is a type of metal poisoning caused by lead in the body. The brain is the most... According to a study published in $2008,1.1 \%$ of the 736 persons consuming wild game meat tested .... the current reference range for acceptable blood lead concentrations in healthy persons ..... Rubin, R. ; Strayer, D.S., eds.
Journals.lww.com Home Sepetember 2016- Volume 95Issue 38 by $\mathrm{CO}$ Marginean 2016-Cited by 1 . Related articles sep 11, 2016- Background : Lead is a toxic element of the environment which leads to major complications once it en. ... Reference. 1. Rubin R, Strayer DS, Rubin E, et al. ... 5th ed. 2008; Philadelphia: Lippincott Williams \& Wilkins, Vol 5.

www.ijhsr.org/IJHSR_Vol.6_May2016/50.pdf by S BathiaCited byl- Related articles Apr 13, 2016-Key words: heavy metals, lead, selenium, mercury, silicon. .... references ... 2008. 29(2): 63-70. Rubin R, Strayer DS. Environmental. 